Maíra M. Garcia*, Tiago R. Oliveira, Daniel Papoti, Khallil T. Chaim, Maria C. G. Otaduy, Daniel Erni, and Waldemar Zylka

\title{
Experimental and numerical investigations of a small animal coil for ultra-high field magnetic resonance imaging (7T)
}

https://doi.org/10.1515/cdbme-2019-0132

\begin{abstract}
The purpose of this work was to develop and investigate a radiofrequency (RF) coil to perform image studies on small animals using the 7T magnetic resonance imaging (MRI) system, installed in the imaging platform in the autopsy room (Portuguese acronym PISA), at the University of São Paulo, Brazil, which is the unique 7T MRI scanner installed in South America. Due to a high demand to create new specific coils for this $7 \mathrm{~T}$ system, it is necessary to carefully assess the distribution of electromagnetic (EM) fields generated by the coils and evaluate the patient/object safety during MRI procedures. To achieve this goal 3D numerical methods were used to design and analyse a 8-rungs transmit/receive linearly driven birdcage coil for small animals. Calculated magnetic field $\left(B_{1}\right)$ distributions generated by the coil were crosschecked with measured results, indicating good confidence in the simulated results. Electric field results were post-processed and predictions of local specific absorption rate (SAR) values were achieved for a spherical phantom filled with muscle-like tissue, indicating that the sample would not suffer any unsafe deposition of energy. Post mortem abdomen images obtained from a rat presented good image quality and no artifacts related to field non-homogeneity were observed.
\end{abstract}

*Corresponding author: Maíra M. Garcia, Faculty of Electrical Engineering and Applied Natural Sciences, Westphalian University, Neidenburger Straße 43, Campus Gelsenkirchen, Germany, and General and Theoretical Electrical Engineering (ATE), Faculty of Engineering, University of Duisburg-Essen, Bismarck Straße 81, Campus Duisburg, Germany, e-mail: maira.martins-garcia@stud.uni-due.de

Tiago R. Oliveira, Daniel Papoti, Department of Biomedical Engineering, Federal University of $\mathrm{ABC}$, Campus São Bernardo do Campo, Brazil

Khallil T. Chaim, Maria C. G. Otaduy, Department and Institute of Radiology, University of São Paulo, Campus São Paulo, Brazil Daniel Erni, General and Theoretical Electrical Engineering (ATE), Faculty of Engineering, University of Duisburg-Essen, and CENIDE - Center for Nanointegration Duisburg-Essen, Duisburg, Germany

Waldemar Zylka, Faculty of Electrical Engineering and Applied Natural Sciences, Westphalian University, Campus Gelsenkirchen, Germany
Keywords: ultra-high field MRI, electromagnetic simulation, small animal RF coil, SAR, MRI safety.

\section{Introduction}

Magnetic resonance imaging is a non-invasive and nonionizing imaging technique, which uses energy in the radiofrequency range. The transmission and receiving of the RF signal during a MRI procedure are done through RF coils. Transmission coils (Tx) are responsible for the generation of an oscillating magnetic field $\left(B_{1}\right)^{1}$ perpendicular to the static magnetic field $\left(B_{0}\right)$ that interacts with the sample. Receive coils $(\mathrm{Rx})$ have the capability to receive EM radiation produced by the sample in response to the $B_{1}$ field application [1].

Therefore, during MRI procedures a patient's body can absorb RF energy, due to the electromagnetic response of the patient tissue. The excessive energy absorbed may result in tissue heating, which can be significant for systems that extensively use high $B_{1}$ fields [2]. Usually, patient safety is assessed through the dosimetric term SAR. For ultra-high fields $\left(B_{0} \geq 7 \mathrm{~T}\right)$, electrical and magnetic components of RF fields, as well as the SAR profile in the object imaged, are highly complex and spatially non-uniform. And to overcome these challenges, RF coils can be designed specifically for different body regions and operations, ensuring a specific EM field distribution and consequently controlling the deposition of EM energy in tissues. The prediction of RF field distribution, as well as the estimation of SAR and temperature increase in the patient, are normally based on 3D numerical simulations using advanced software tools.

In this study, a transmit/receive linearly driven birdcage coil for $B_{0}=7 \mathrm{~T}$ was designed and constructed to investigate small animals, like rats and mice. Experimental and numerical evaluations were done in order to investigate the incident RF magnetic field distribution and to ensure animal safety when using the designed coil in 7T MRI procedures.

1 For convenience in this paper we use the term magnetic field for the magnetic flux density. 


\section{Materials and Methods}

\subsection{Design, modelling and construction of the RF coil}

The RF coil was designed for the 7T MRI scanner (Siemens Healthcare, Germany) installed at PISA platform in Brazil.

The initial process of geometry development and component values selection was made using the Birdcage Builder (Center for NMR Research, USA) [3], and its results were used for modelling the complete coil structure with COMSOL Multiphysics 5.4 (COMSOL Inc, Sweden), in order to perform 3D numerical analysis. A 8-rungs transmit/receive linearly driven high-pass birdcage coil was selected after the previous analysis.

Figure 1 presents the geometry of the designed coil. It was built using copper strips as conductors (thickness $t=0.07 \mathrm{~mm}$ ), placed on a polyvinyl chloride (pvc) cylinder (length $l_{\mathrm{pvc}}=$ $220 \mathrm{~mm}$, external diameter $d_{\mathrm{pvc}}=102 \mathrm{~mm}$ ). The 8 rungs (length $l_{\text {rung }}=138 \mathrm{~mm}$, width $w_{\text {rung }}=6.4 \mathrm{~mm}$ ) are connected at theirs extremities to 2 end-rings, which presents 8 gaps each $(1 \mathrm{~mm})$, where capacitors are placed. 15 capacitors $C=4.3 \mathrm{pF}$ and one variable capacitor $C_{v}=5-15 \mathrm{pF}$, placed $180^{\circ}$ apart from the excitation port, were used. The end-ring's width is $w=13.2 \mathrm{~mm}$.

To avoid EM interferences from other hardware components of the MRI scanner, an EM shielding apparatus was designed to be connected to the coil. It consists of 8 copper sections equally spaced at the external surface of a pvc cylinder (height $h_{\text {shield }}=210 \mathrm{~mm}$, external diameter $d_{\text {shield }}=150 \mathrm{~mm}$ ), separated by $1 \mathrm{~mm}$ gaps, where 32 capacitors $C_{\text {shield }}=1000 \mathrm{pF}$ are distributed and connected.
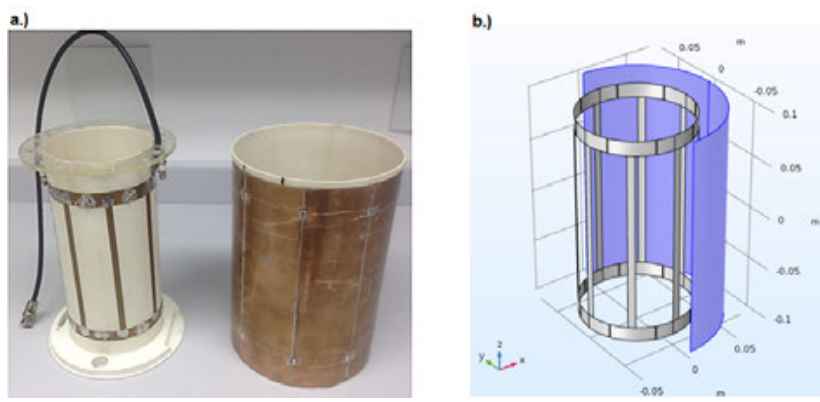

Fig. 1: a.) Birdcage coil and EM shield constructed. b.) Birdcage coil geometry modelled using COMSOL.

The coil has a tuning and matching network to ensure optimal coil's performance. The matching circuit consists of 2 capacitors $C_{\mathrm{m}}=8.2 \mathrm{pF}$, connected in series at the supply point, and the tuning is performed using a variable capacitor $C_{\mathrm{t}}=1$ $10 \mathrm{pF}$, placed on the opposite side of the matching circuit.

\subsection{Numerical investigations}

After modelling the birdcage coil using COMSOL, two spherical phantoms of diameter $d_{p}=70 \mathrm{~mm}$ were created to load the coil during the simulations. The first one is a phantom filled with water. It was used to analyse the $B_{1}$ field distribution inside the sample, for later comparison with the experimental results, intending to validate the methodology developed for the simulations.

The second one was filled with muscle-like tissue to evaluate the sample safety during MRI procedures. The sample safety was assessed through the calculation of the local SAR values, which is the dosimetric term used to describe the absorption of RF radiation. SAR calculations were done using the magnitude of the electric field $(|\vec{E}(\vec{r})|)$ and post-processing of the simulation results, by solving:

$$
\mathrm{SAR}=\frac{1}{V} \int_{V} \frac{\sigma(\vec{r})}{2 \rho(\vec{r})}|\vec{E}(\vec{r})|^{2} d V
$$

where $\sigma$ is the specific tissue electrical conductivity and $\rho$ the tissue density.

The electrical conductivity of tissues is frequency dependent. Therefore, for SAR calculation was assumed the muscle conductivity at $300 \mathrm{MHz}$ as $\sigma=0.77 \mathrm{~S} / \mathrm{m}$ [4]. The value of $\rho=1090 \mathrm{~kg} / \mathrm{m}^{3}$ was used for tissue density. Finally, to perform all simulations, the excitation's port values were assigned using the values obtained at experimental tests, and local mesh refinement was applied.

\subsection{Experimental investigations}

To perform the first test, a cylindrical sample made of pvc, which has an internal sphere of diameter $d_{\exp }=70 \mathrm{~mm}$ filled with water and a solution of $1 \mathrm{~g} / \mathrm{L}$ of $\mathrm{NaCl}$ and $2 \mathrm{~g} / \mathrm{L}$ of $\mathrm{CuSO}_{4}$, was positioned at the isocenter of the $\mathrm{RF}$ coil.

In order to obtain $B_{1}$ field maps, the pulse sequence SA2RAGE was used [5]. The necessary parameters were $\mathrm{TR}=2400 \mathrm{~ms}$ and $\mathrm{TE}=1.24 \mathrm{~ms}$ [6]. The maximum signal amplitude was obtained using $20 \mathrm{~V}$ for the applied Tx voltage. A total of 56 slices were acquired in coronal, sagittal and axial directions.

The validation was done comparing the simulated and experimental $B_{1}$ field distributions. To quantify the homogeneity of the coil for both simulated and experimental results, the parameter non-uniformity (NU) was used [7]. It was analysed inside a region of interest (ROI) with diameter $d_{\mathrm{ROI}}=52.1 \mathrm{~mm}$ (approximately $74.4 \%$ of the phantom's size). The percentage value of NU was calculated as $\mathrm{NU}=\frac{s}{B_{m}} 100$, where $s$ is the standard deviation related to the mean value of the magnetic field $\left(B_{m}\right)$. 
Post mortem images were also obtained for image quality evaluation of the designed coil and to analyse the presence of artifacts related to $B_{1}$ field inhomogeneities. A post mortem rat was used for this purpose. Images of the animal's abdomen were obtained using a 3D FLASH sequence, that is based on gradient-echo sequence, using $\mathrm{TR}=9 \mathrm{~ms}$, $\mathrm{TE}=4.1 \mathrm{~ms}$, flip an$\mathrm{gle}=15^{\circ}$, and $\mathrm{FOV}=212 \mathrm{~mm} \times 79.5 \mathrm{~mm}$. Coronal images of 112 slices were done.

\section{Results and discussion}

\section{1 $B_{1}$ field distribution}

The calculated $B_{1}$ field distribution for the empty coil can be seen in Fig. 2. The $B_{1}$ field distribution was achieved at the resonance frequency for this structure: $298,7 \mathrm{MHz}$ (obtained after a modal analysis of the structure of the coil), using the variable capacitor value as $C_{v}=5 \mathrm{pF}$.

The calculated magnetic field generated by this coil presented a very homogeneous and symmetrical internal distribution, especially for a ROI comparable with a small animal body (approximately 70mm diameter and 100-120mm length). These results ensure that the designed RF birdcage coil is suitable for obtaining good quality images for small animals.
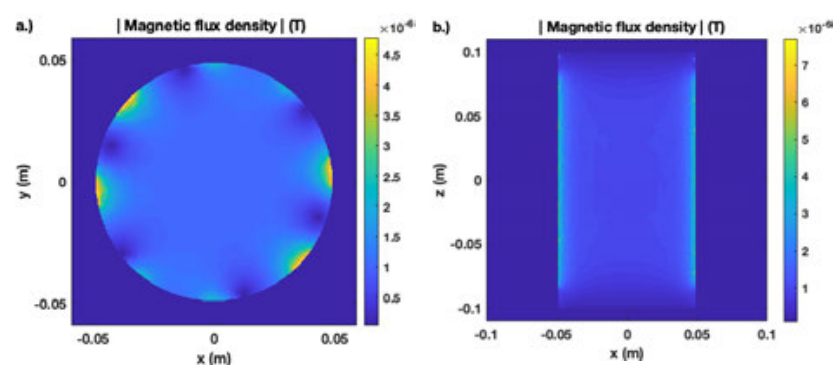

Fig. 2: Magnetic flux density simulated inside the coil, observed on sections: a.) axial $(z=0)$ and b.) coronal $(y=0)$.

Magnetic field distributions inside the spherical water phantom were also experimentally measured and are compared with the simulated results for the water phantom in Fig. 3 . For both measured and calculated results, the voltage of $20 \mathrm{~V}$ for the coil's excitation value was used. For comparison, all the maps obtained were normalized in relation to the central value of each section: axial, sagittal and coronal.

A quantitative analysis of the homogeneity of $B_{1}$ distributions was performed by using the NU parameter. The ROI was delineated at the axial plane of the experimental result (Fig. 3a) and was defined as the maximal internal region of
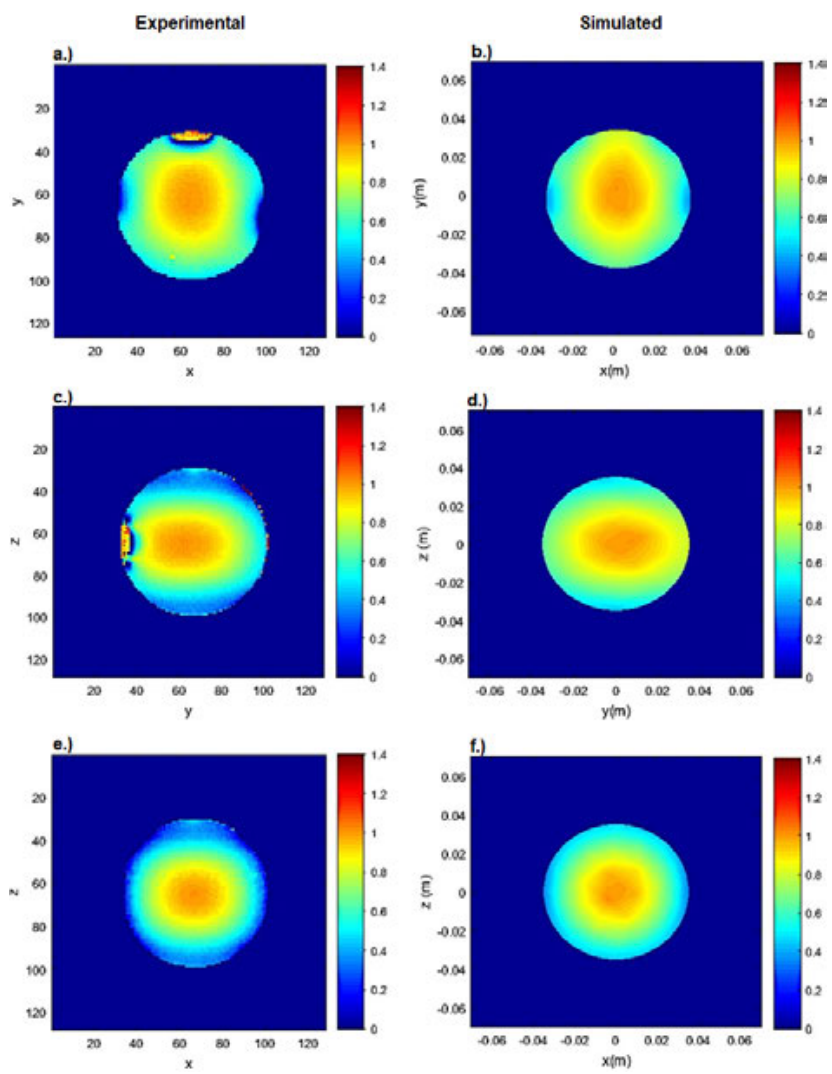

Fig. 3: Magnetic field distributions analysed inside the spherical water phantom. Experimental results are presented for the a.) axial, c.) sagittal and e.) coronal planes. Calculated maps are also presented for the b.) axial, d.) sagittal and f.) coronal sections. In a.) and c.) a bubble is observed.

the sample avoiding the bubble. The NU parameter for the numerical and experimental $B_{1}$ maps were $\mathrm{NU}_{\text {num }}=6.68 \%$ and $\mathrm{NU}_{\exp }=6.76 \%$, respectively. The small difference between them indicates good confidence.

\subsection{Post mortem images aquisition}

Figure 4 presents coronal slices at different positions from a post mortem rat's abdomen. Even though the signal-to-noise ratio (SNR) was not optimum, the images show no artifact due to $B_{1}$ field non-uniformities.

The rat's extremities were outside the coil, which means that the length of the rat (along the z-axis) was bigger than the homogeneous $B_{1}$ field region, as presented in Fig. 2b. Outside the homogeneous region, the $B_{1}$ magnitude decays substantially, and it is not enough to excite the spins (smaller flip angle than in the center of the coil). Thus, it explains the loss of image quality at the rat's extremities in Fig. 4. 

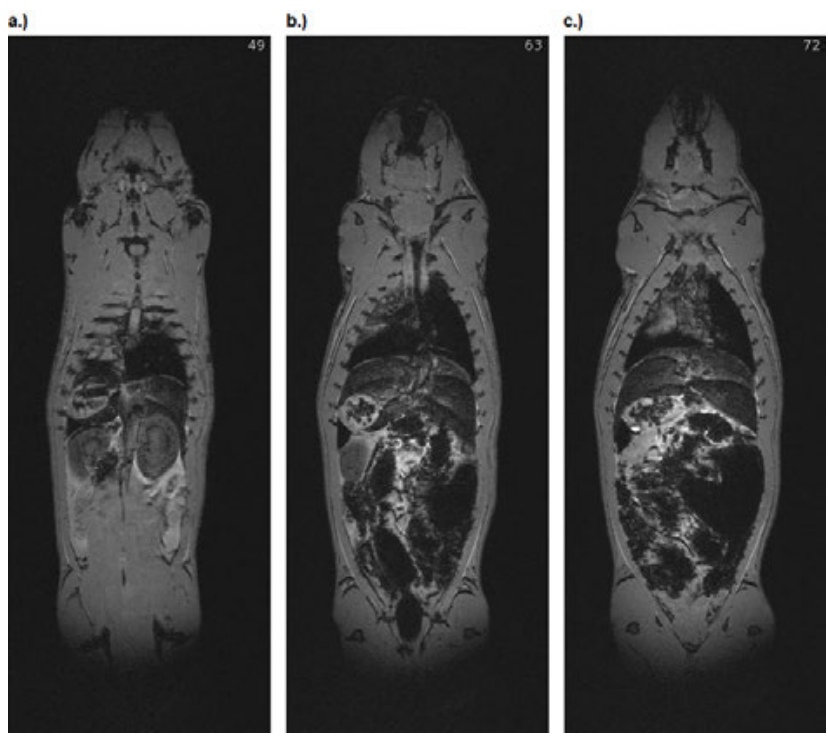

Fig. 4: Post mortem coronal images of the rat at 3 different positions along y axis.

\subsection{SAR predictions}

Using the software COMSOL, the $70 \mathrm{~mm}$ muscle-like tissue spherical phantom was placed at the coil's center. It was created in order to represent the head of a small animal submitted to a MRI procedure. Estimations of the RF deposition inside the phantom are analysed in Fig. 5, where the axial and sagittal planes are presented.

According to the FDA (Food and Drugs Administration) and IEC (International Electrotechnical Commission), values higher than $40 \mathrm{~W} / \mathrm{Kg}$ for human body parts achieved during ultra-high field MRI procedures are considered as high risk [8]. For our results, we consider that the animal will be safe, since the phantom will not achieve such high SAR values.
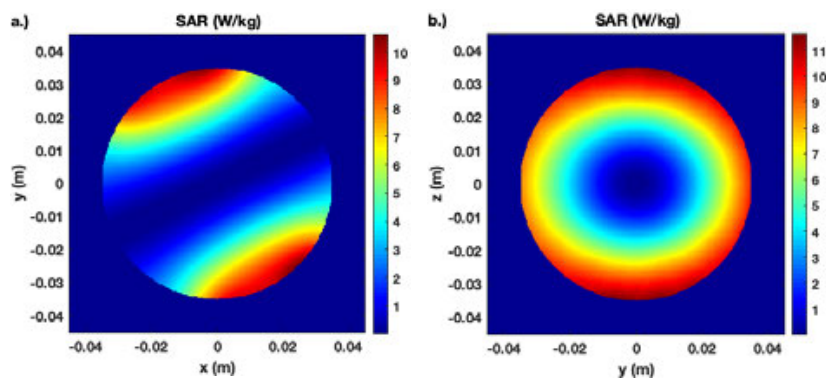

Fig. 5: SAR predictions in a 70mm spherical phantom, filled with muscle. In a.) is the axial view $(\mathrm{z}=0)$ and in $\mathrm{b}$.) the sagittal view $(\mathrm{x}=0)$.

\section{Conclusions and outlook}

The aim of the presented work was to develop a workflow that could permit to help in the traditional process of coil design and analysis, and especially, to ensure patient safety when using a designed coil in 7T MRI procedures at PISA. The numerical and experimental investigation made in this work show that the proposed birdcage coil allows performing satisfactory image studies on small animals, with reasonable confidence that the animal will be safe, due to the SAR results obtained. It is intended to improve the simulation and validation processes, using more realistic phantoms to evaluate patient's SAR distributions, and to measure the patient's temperature, which can be simulated and validated in the future.

\section{Author Statement}

Research funding: Research reported in this publication was supported by the Coordination for the Improvement of Higher Education Personnel (CAPES): Full PhD Program Abroad, process n. 88881.173609/2018-01. Conflict of interest: Authors state no conflict of interest. Ethical approval: The research related to animal use complies with all the relevant national regulations, institutional policies and has been approved by the authors' institutional review board or equivalent committee.

\section{References}

[1] Jin J. Electromagnetic Analysis and Design in Magnetic Resonance Imaging. 1st ed. Florida: CRC Press;1999.

[2] Moros EG. Physics of thermal therapy - Fundamentals and clinical applications. 1st ed. Florida: CRC Press;2013.

[3] Chin CL, Collins CM, Li S, Dardzinski BJ, Smith MB. Bircagebuilder: Design of specified-geometry birdcage coils with desired current pattern and resonant frequency. Concepts Magn. Reson. 2002;15:156-163.

[4] Gabriel S, Lau R, Gabriel C. The dielectric properties of biological tissues: II. Measurements in the frequency range $10 \mathrm{~Hz}$ to $20 \mathrm{GHz}$. Phys. in Med. Bio 1996;41:2251-2269.

[5] Marques JP, Kober T, Krueger G, Van der Zwaag W, Van de Moortele PF, Gruetter R. MP2RAGE, a self bias-field corrected sequence for improved segmentation and $T_{1}$-mapping at high field. Neurolmage 2010;49:1271-1281.

[6] Eggenschwiler F, Magill AW, Kober T, Gruetter R, Marques JP. Sa2RAGE - A new sequence for rapid 3D $B_{1}{ }^{+}$-mapping with a wide sensitivity range. 18 Proc. Soc. Mag. Reson. Med. 2010;2843.

[7] Papoti D, Vidoto ELG, Martins MJ,Tannús A. RF transmitter/receiver coils with passive decoupling for MRI experiments on small animals. Rev Bras Fis Med 2010;4:49-51.

[8] Fiedler T, Ladd M, Bitz A. SAR simulations and safety. Neurolmage 2018;168:33-59. 